\title{
Assessing the Impact of Climatic Variables on Malaria Cases among Pregnant Women in South-Western Nigeria
}

\author{
Babajide Sadiq", Perry Brown \\ Department of Epidemiology and Biostatistics, Institute of Public Health, Florida A\&M University, 1601 S Martin Luther King, USA
}

Copyright $(2017$ by authors, all rights reserved. Authors agree that this article remains permanently open access under the terms of the Creative Commons Attribution License 4.0 International License

\begin{abstract}
The purpose of this study was to determine the effect of climatic factors on malaria cases among pregnant women in Ogun State. A zero-truncated negative binomial regression was used to analyze the effect of meteorological variables and malaria cases among pregnant women using a 10 year historical data from January 2004 to December 2013. A bivariate analysis shows an association between rainfall $((0.000935), 95 \% \mathrm{CI}$ 0.00026-0.00161, p-value 0.0070), humidity ((0.007698), 95\% CI 0.00054-0.01486, p-value 0.0353), and maximum temperature ((-0.04628), 95\% CI -0.08037- -0.01219, p-value 0.0082) and malaria cases among pregnant women. There is a significant relationship between meteorological variables and malaria cases among pregnant women in Ogun State, Nigeria.
\end{abstract}

Keywords Zero-truncated Negative Binomial, Meteorological Variables, Malaria

\section{Introduction}

\subsection{Malaria in Pregnant Women}

Malaria infection during pregnancy is a major public health problem in tropical and subtropical regions throughout the world. In endemic areas such as Africa, pregnant women are 3 times more likely to suffer from severe malaria infection and have a death rate of $50 \%$ when comparing to non-pregnant women [1]. The severity of malaria infection among pregnant women is more likely to be in the second trimester [2]. It is assumed that the majority of malaria infections during pregnancy results from two main factors: the immunocompromised state of pregnancy and placental sequestration of infected erythrocytes. Adults who live in malaria endemic regions acquire some immunity to malaria infection as a result of immunoglobulin production during prior infections in childhood. This immunity diminishes significantly in pregnancy, particularly in primigravidas [3]. Fig. 1 shows the mechanisms of malaria infection in pregnant women.

A study conducted among 300 women in rural Ghana found a higher rate of anemia, clinical malaria, and placental burden of infection among primigravidas compared with multigravidas [4]. Splenic sequestration of malaria infected erythrocytes leads to folic acid deficiency and microcytic anemia in adults. In pregnant women, additional sequestration of malaria-infected erythrocytes occurs in the placenta thereby causing an excessive and severe anemia as a result of the infection. Interestingly, the greatest degree of placental infestation is seen in women who have the highest level of immunity, leading to milder maternal symptoms but a disproportionate increase in fetal complications [1]. According to the Nigeria Malaria Control Program Strategic Plan 2009-2013, malaria contributed to $11 \%$ of maternal mortality and $10 \%$ of low birth weight annually in Nigeria $[5,6]$. The aim of this study is to examine the relationship between climatic factors and malaria cases among pregnant women in Ogun State. 


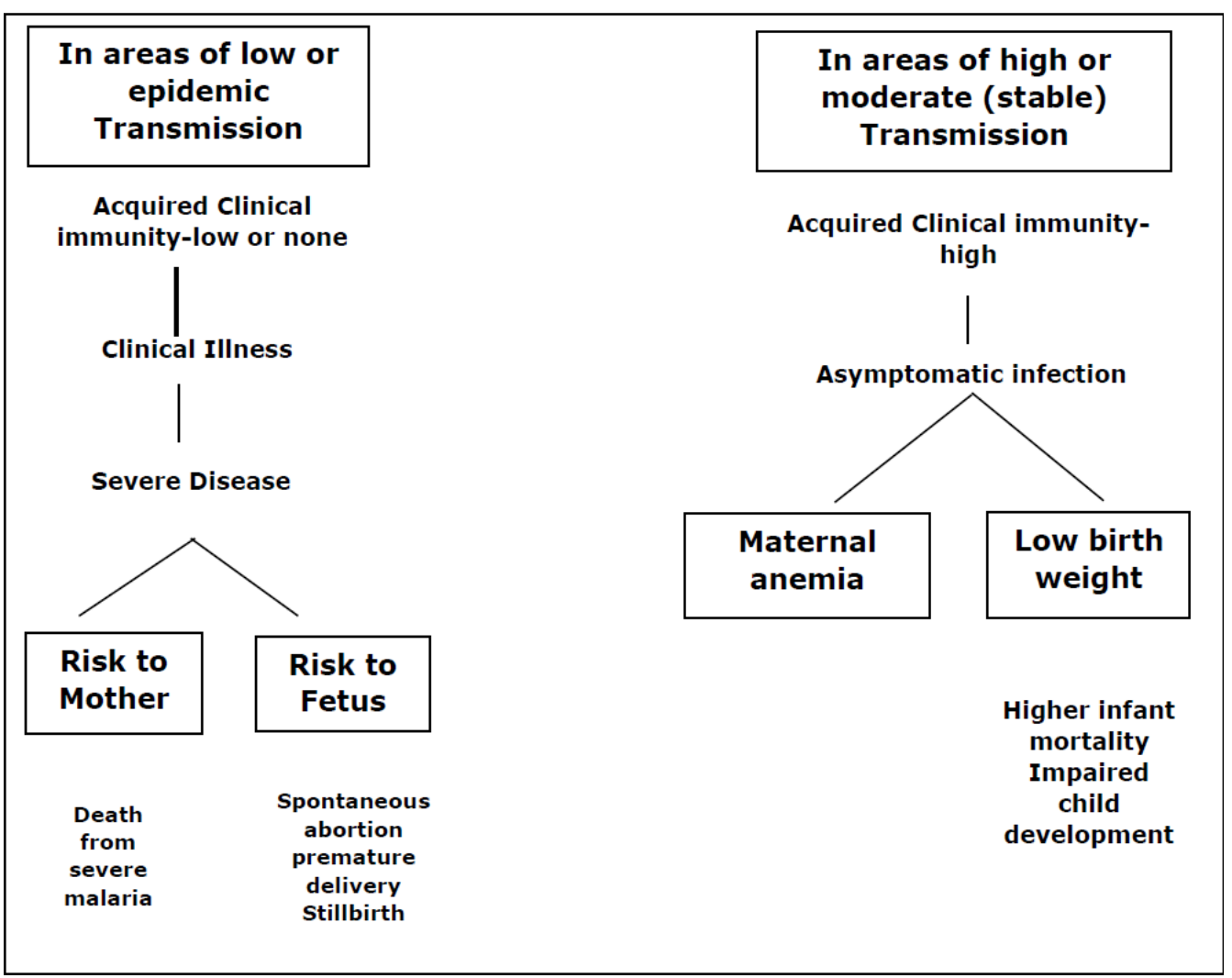

Figure 1. Malaria during Pregnancy

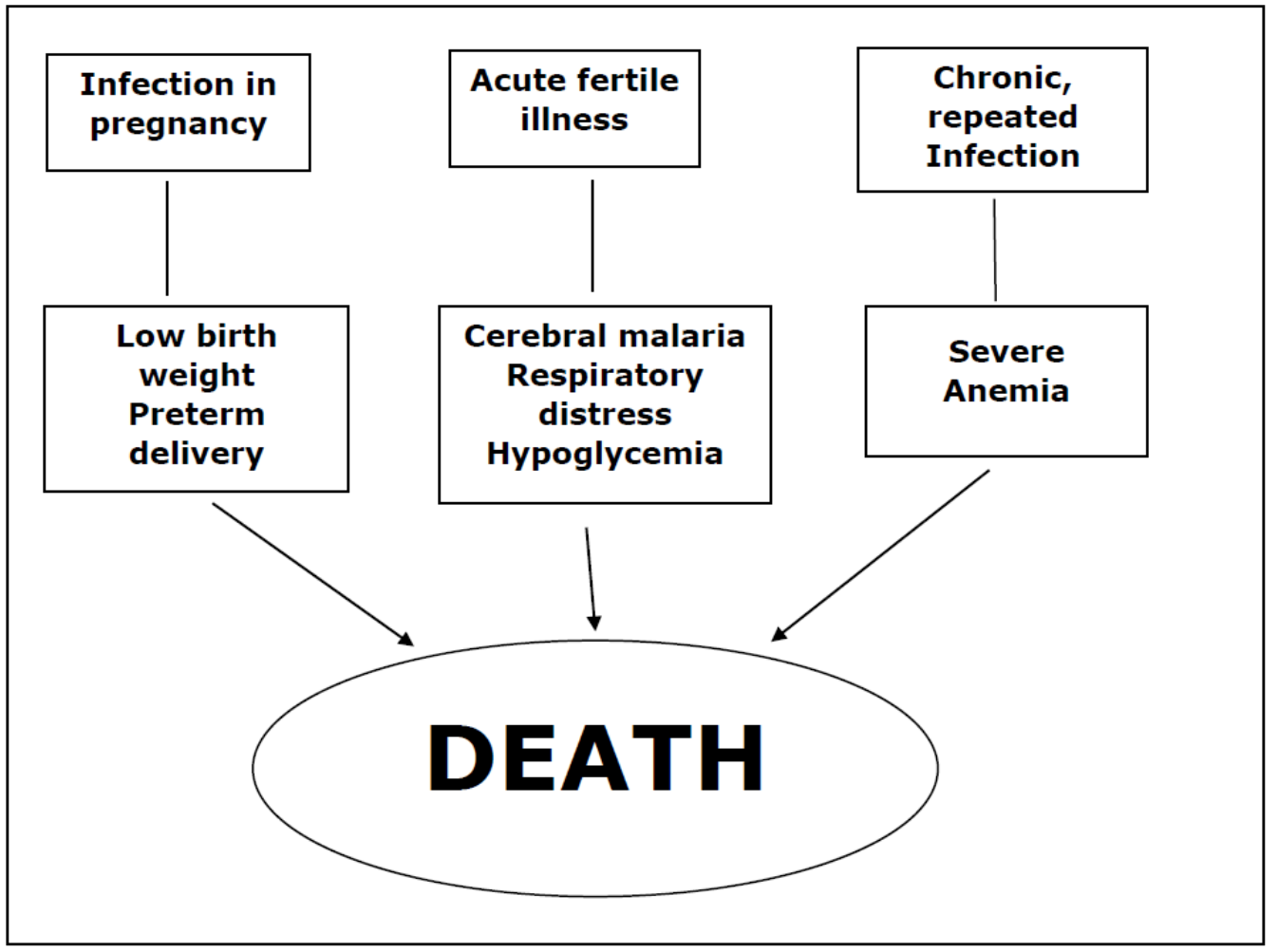

Figure 2. Different ways in which malaria kills children

\subsection{Malaria in Children}

Malaria is the third biggest killer of children globally. In 2003 , more than 3000 children died every day from malaria in Africa [7]. In fact, a child somewhere in the world dies every minute of malaria and African children are killed every 30 seconds. It is also estimated that $86 \%$ of total 
malaria deaths in the world were in children under five years of age $[8,9]$. Most deaths occurred among children in Africa. In 2014, it was estimated that a child died every minute from malaria and about 460000 African children died before their fifth birthday [10]. According to the Nigeria Malaria Control Program Strategic Plan 2009-2013, malaria is the leading cause of death in children under five accounting for 225,000 deaths in Nigeria [6]. Fig. 2 shows the different ways in which malaria kills children. There are three principal mechanisms. The first is low birth weight due to infection in pregnant women. This is the major risk factor for death during the first month of life. Second, an acute infection which may be presented as seizures and coma (cerebral malaria) may kill a child directly and quickly. The third is repeated malaria infection which could progress to severe malaria and substantially increases the risk of death [3].

\subsection{Study Location}

Ogun State is located at the south-western part of Nigeria. The population as of the 2011 Census was $4,397,604$. There were $2,186,301$ males and 2,211,303 females [11]. The land area of the state is about $6,335.7$ square miles $(16,409$ square kilometers). Ogun State is located in a moderately hot, humid tropical climatic zone of southwest part of Nigeria. There are two distinct seasons in the state namely, the rainy season which from March/April to October/November and the dry season which lasts for the rest of the year, October/November till March/April. The temperature is relatively high during the dry season with a mean temperature of around $30^{\circ} \mathrm{C}$. The harmattan, brought in the northeasterly winds from December February, has ameliorating effects on the dry season high temperatures. Low temperatures are experienced during the rains, especially between July and August when the temperatures could be as low as $24^{\circ} \mathrm{C}$. The distribution of rainfall varies from about $1000 \mathrm{~mm}$ in the western part to about $2000 \mathrm{~mm}$ in the eastern part [12]. Fig. 3 shows the map of Ogun State with its local government areas.

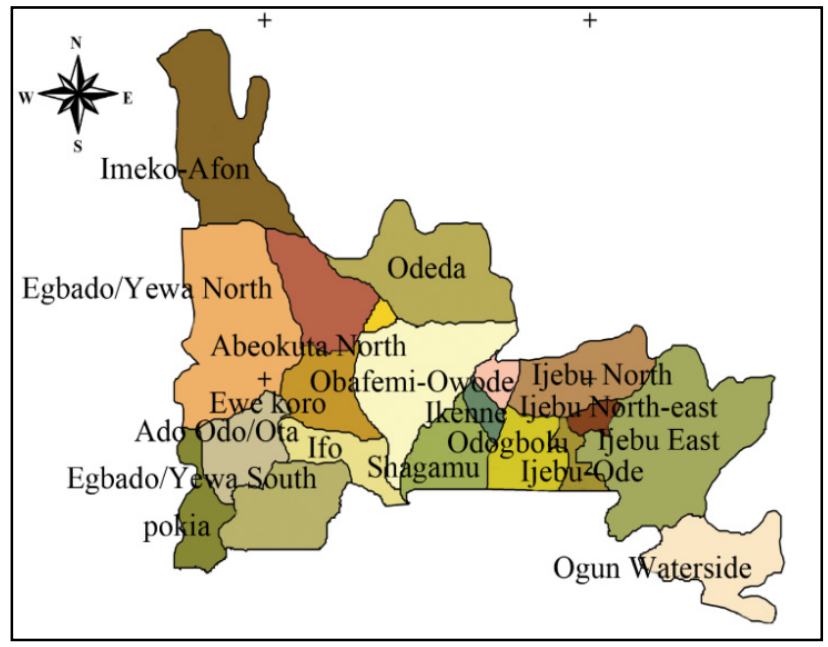

Figure 3. Map of Ogun State, Nigeria

\section{Materials and Methods}

\subsection{Data Collection and Management}

A historical monthly data for both malaria cases and climatic variables for Ogun State from 2004-2013 was used for this study. Malaria data was abstracted from the monthly health directorate data from 2004-2013 for Ogun State. This health malaria surveillance database is maintained by the Ogun State Ministry of Health. The malaria data was received from the primary, secondary and tertiary clinics and hospital from all the 20 local government of the state. This data consists of malaria cases and deaths for all people less than 5 years, 5-14 years and greater than 15 years and malaria cases and deaths for pregnant women. Cases were classified based on severity: 1) Uncomplicated cases: which is the malaria attack that lasts for 6-10 hours and is considered an outpatient case, 2) Severe cases: in which infections are complicated by serious organ failures or abnormalities in the patient's blood or metabolism and are inpatients. After receiving the data from the hospitals and clinics, the data entry manager calculates the cases by clinic and entered them into the malaria database using the Microsoft Excel software. Reports were also generated using this software. This report is computed and sent to the national malaria surveillance system in Abuja, Nigeria.

The meteorological data from 2004-2013 was collected from the Nigeria Meteorological Agency. This has the average monthly rainfall, monthly mean relative humidity, and monthly mean minimum and mean maximum temperature. This data was received from the national office from each weather station across the country. The data was used to generate a report in the national climatic database using Microsoft Excel. There are two weather stations in Ogun State, one in Abeokuta the state capital and Ijebu Ode another major city in the state. These cities are about $89 \mathrm{~km}$ ( 55 miles) apart from each other.

The maximum temperature is the average of the overall maximum temperatures measured on each day (which is usually in the afternoon). For example for January, the maximum temperature for each day divided by 31 ). The same procedures used for maximum temperature were employed to calculate monthly averages for the minimum temperature and average humidity. The monthly rainfall is different because some days may have no rainfall (zero rainfall), therefore, rainfall for the month was noted as the total rainfall for the month rather than the average, (e.g. if the January value is 200 , this is the total rainfall for January 1 to January 31 of that year).

The data collected from the malaria program database, malaria cases, deaths and severity of malaria cases was compiled monthly from 2004-2013. These data were entered into a Microsoft Excel spreadsheet for cleaning. During cleaning, some cases were found to be missing for year 2009 (July-December); while some did not have sufficient records for further analyses. For the missing values the cubic spline interpolation method was used to 
interpolate and assign the missing values. The cubic spline interpolation is a common numerical curve fitting strategy that fits a "smooth curve" to the known data, using cross-validation between each pair of adjacent points to set the degree of smoothing and estimate the missing observation by the value of the spline. Also, due to inconsistencies in reporting, some years did not have the same variables as the other. For example 2007, 2008 and 2009 only reported malaria severity while in 2004; ages were only reported while the severity was missing. In order to make the best use of the dataset and to be able to reach an accurate result as possible, variables with inconsistent records were removed from the final analysis. Overall, the final data includes the monthly malaria cases from 2004-2013 for all ages.

\subsection{Statistical Analysis}

The analysis for this study included descriptive and zero-truncated negative binomial. Zero-truncated negative binomial was used to analyze the relationship between the climatic variables and malaria cases. This model was used because the following assumptions were true about the data:

- The malaria cases are the number of times people are diagnosed with malaria (count) at a particular/fixed time (month).

- The number of malaria cases in a month cannot be zero. (That is there will be no month with no incidence of malaria cases).

- There is over dispersion of malaria cases, the mean of the malaria cases is not equal. (That is there is a significant difference between the mean and the variance). Figure 4 shows over dispersion of the malaria cases.

- The occurrence of malaria cases is independent (That is the occurrence of malaria cases in a month does not affect the probability of the occurrence of malaria cases in another month).

\subsection{Zero-truncated Negative Binomial Regression using Proc NImixed}

In order to use proc nlmixed to perform truncated negative binomial regression, we must supply it with a likelihood function [13].

The probability that an observation has count (y) under the negative binomial distribution (without zero truncation) is given by the equation:

For Over dispersion Parameter:

$$
[P(Y=y)=\{y+\operatorname{frac}\{1\}\{\text { alpha }\}-1
$$

Choose

$$
\begin{gathered}
\text { frac }\{1\}\{\text { alpha }\}-1\} \text { left }(\text { frac }\{1\} \\
\{1+\text { alphamu }\} \text { right })^{\wedge} \\
\{\text { frac }\{1\}\{\text { alpha }\}\} \text { left } \\
\left(\text { frac }\{\text { alphamu }\} \text { right }{ }^{y},\right]
\end{gathered}
$$

Where (alpha) is the over dispersion parameter and ( $\mathbf{m u})$ is the mean of the negative binomial distribution.

\section{With zero truncation:}

We calculate the probability that $(Y=y)$ conditional on $(Y>0)$, that is, that $(Y)$ is observed as $0 \square$ values are not observed.

The probability of a zero count under the negative binomial distribution is

$$
\begin{gathered}
(P(Y=0)=\operatorname{left}(\operatorname{frac}\{1\}\{1+\text { alphamu }\} \text { right }) \wedge \\
\{\operatorname{frac}\{1\}\{\text { alpha }\}\})
\end{gathered}
$$

The conditional probability is then:

$$
\begin{aligned}
& {[P(Y=y) \backslash Y>0)=\operatorname{frac}\{P Y=y)\{P(Y>0)\}} \\
& =\operatorname{frac}\{P(Y=y)\}
\end{aligned}
$$

Choose

$$
\begin{gathered}
\text { frac }\{1\}\{\text { alpha }\}-1\} \text { left }(\text { frac }\{1\} \\
\{1+\text { alphamu }\} \text { right })^{\wedge}\{\text { frac }\{1\} \\
\{\text { alpha }\} \text { left }(\text { frac }(\text { alphamu }\} \\
\{1+\text { alphamu }\} \text { right })^{\wedge} y \text { frac }\{1\} \\
\{1-\text { left }(\text { frac }\{1\} \\
\{1+
\end{gathered}
$$

alphamu $\}$ right $)^{\wedge}\{\operatorname{frac}\{1\}$ (alpha $\left.\left.\left.\}\right\}\right\}.\right]$ frac $\{1\}\{$ alpha $\left.\}-1\right\} \operatorname{left}(\operatorname{frac}\{1\}\{$ alphamu $\}$ right $)^{\wedge}\{\operatorname{frac}\{1\}\{$ alpha $\}\} \operatorname{left}(\operatorname{frac}($ alphamu $\}\{1+$ alphamu $\}$ right $)^{\wedge}$ yfrac $\{1\}\{1-\operatorname{left}(\operatorname{frac}\{1\}\{1+$ alphamu $\}$ right $)^{\wedge}\{\operatorname{frac}\{1\}$ (alpha $\left.\left.\}\right\}\right\}$.]

In negative binomial regression, we model $(\log (m u))$,

The log of the mean (expected counts), as a linear combination of a set of predictors:

$$
\begin{gathered}
{\left[\log (m u)=\text { bet }_{0}+\text { beta }_{1} \times_{1}+\text { beta_ } 2 \times \_2+\right.} \\
\text { beta_3 } \left.\times \_ \text {_ }\right]
\end{gathered}
$$

We supply the last two equations to proc nlmixed to model our data using a zero-truncated negative distribution. 


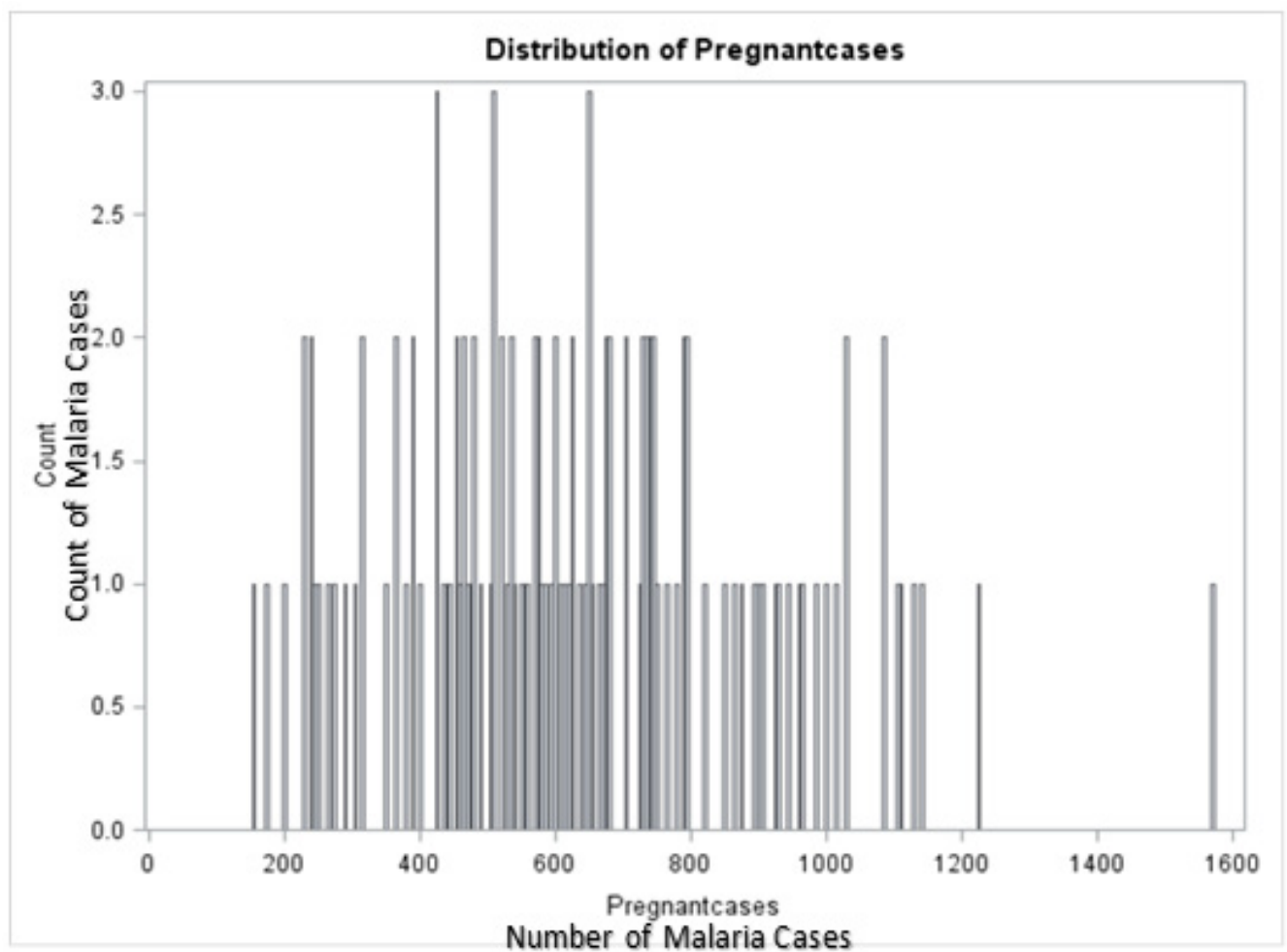

Figure 4. Distribution of malaria cases for pregnant women.

\subsection{Model Building}

Six different types of model were built to analyze the association between malaria cases and meteorological variable. These models were:

Model 1: Malaria Cases $=$ Rainfall

Model 2: Malaria Cases $=$ Humidity

Model 3: Malaria Cases $=$ Minimum Temperature

Model 4: Malaria Cases $=$ Maximum Temperature

Model 5: Malaria cases $=$ Rainfall + Humidity +

Minimum Temperature + Maximum Temperature

Model 6: Malaria cases $=$ Rainfall + Humidity + Maximum Temperature (Significant variables)

All the analyses were performed using $\mathrm{SAS}{ }^{\circledR}$ Version $9.3[14]$.

\section{Results}

\subsection{Malaria Cases}

A total of 75,632 malaria cases were reported for pregnant women in Ogun State, Nigeria from January 2004 to December 2013. This is about $4 \%$ of the total number of malaria cases for this period. With an average of 630 cases per month, this ranges from 154 cases to 1571 cases. Fig. 5 shows the variation of monthly cases from January 2004 to December 2013.

\subsection{Maximum Temperature}

The average maximum temperature was 32.3 degree centigrade. The maximum temperature ranges from 28.3 in July, 2013 to 36.8 degrees centigrade in February, 2010. Fig. 6 shows the variation of maximum temperature from January 2004 to December 2013.

\subsection{Minimum Temperature}

The average minimum temperature was 23.3 degree centigrade. The minimum temperature ranges from $18.1 \mathrm{in}$ January, 2008 to 26.2 degrees centigrade in April, 2010. Fig. 7 shows the variation of minimum temperature from January 2004 to December 2013. 


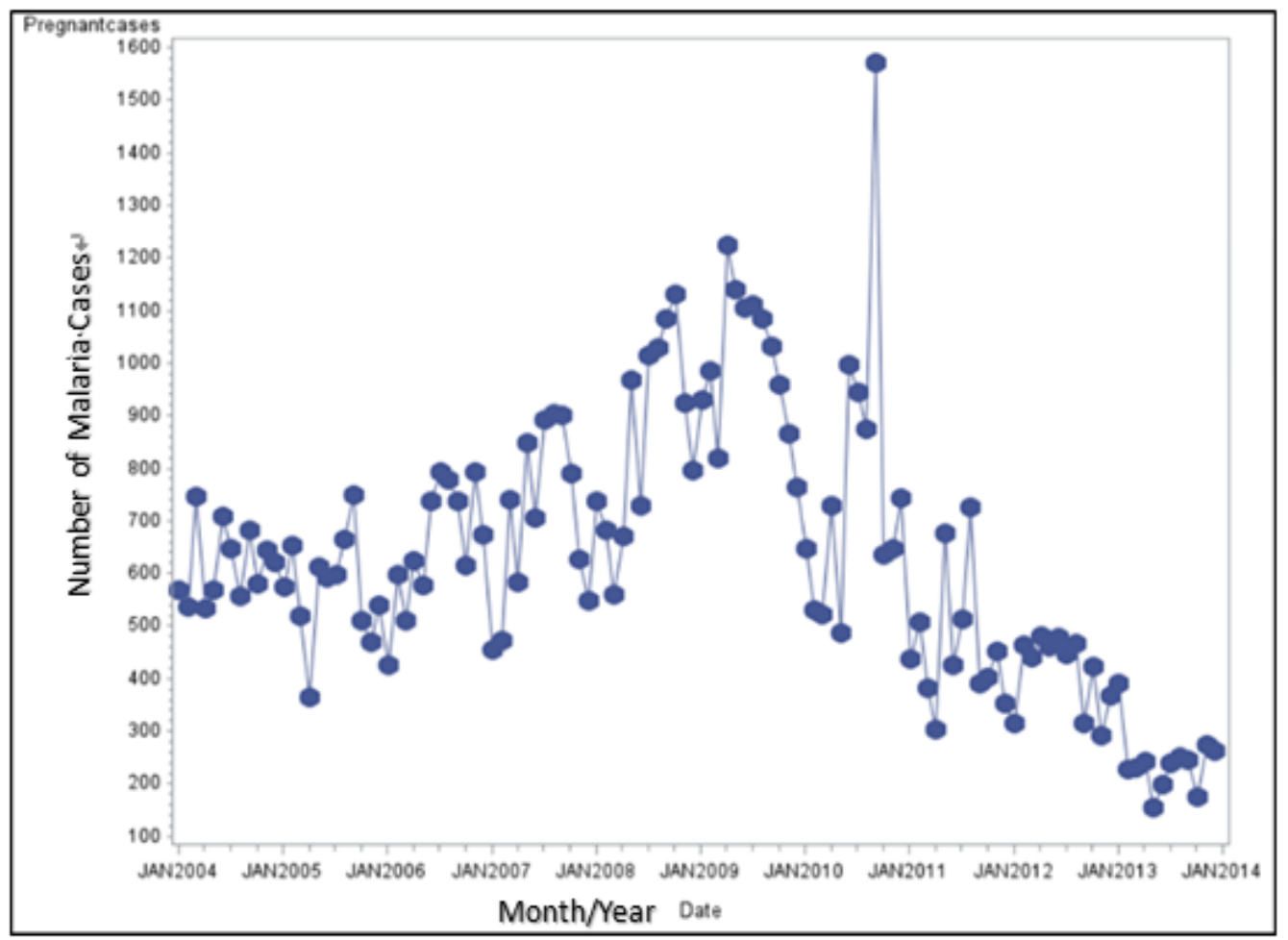

Figure 5. Monthly pregnancy malaria cases between January 2004 and December 2013.

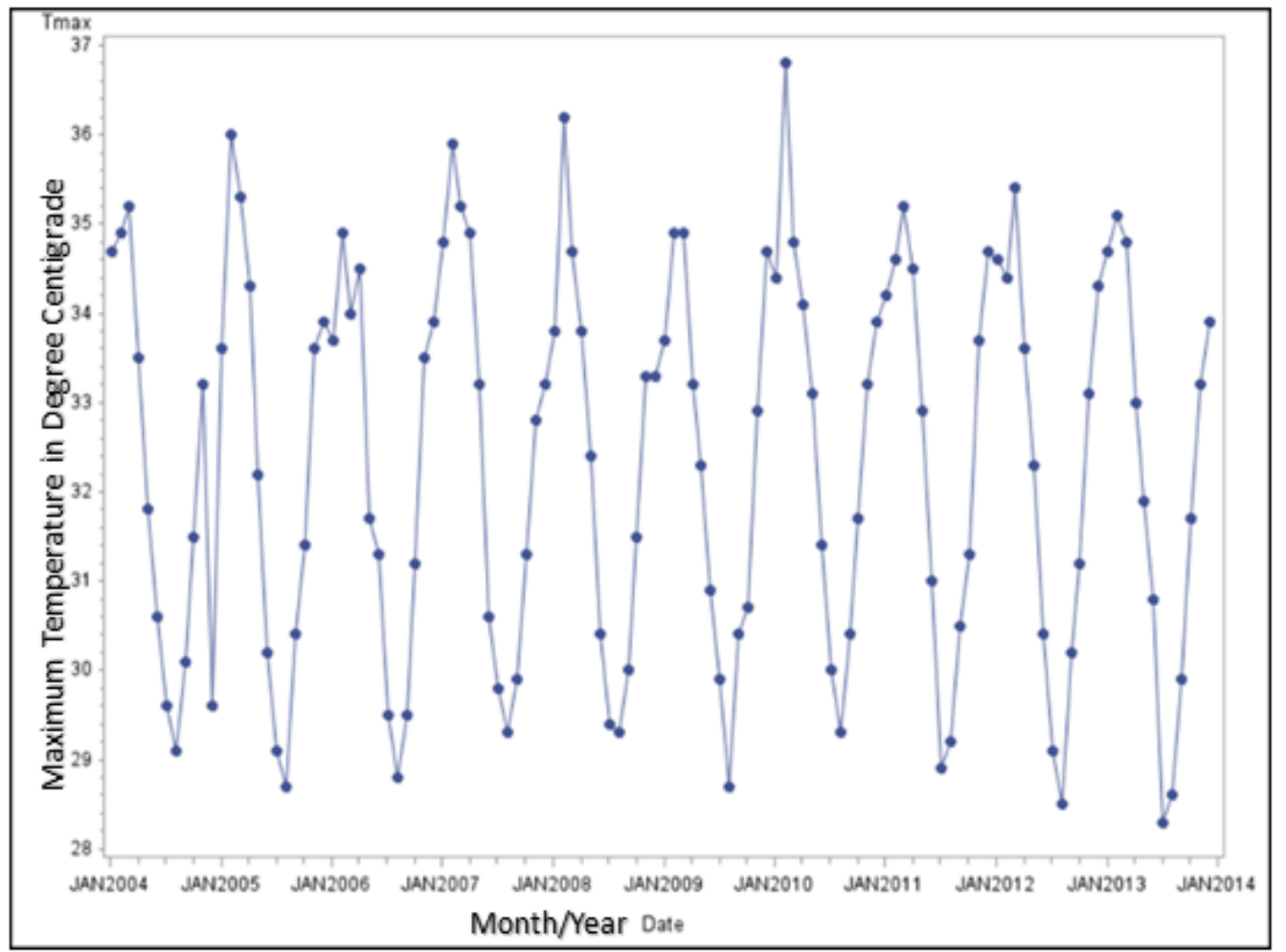

Figure 6. Monthly maximum temperature in degree centigrade between January 2004 and December 2013 


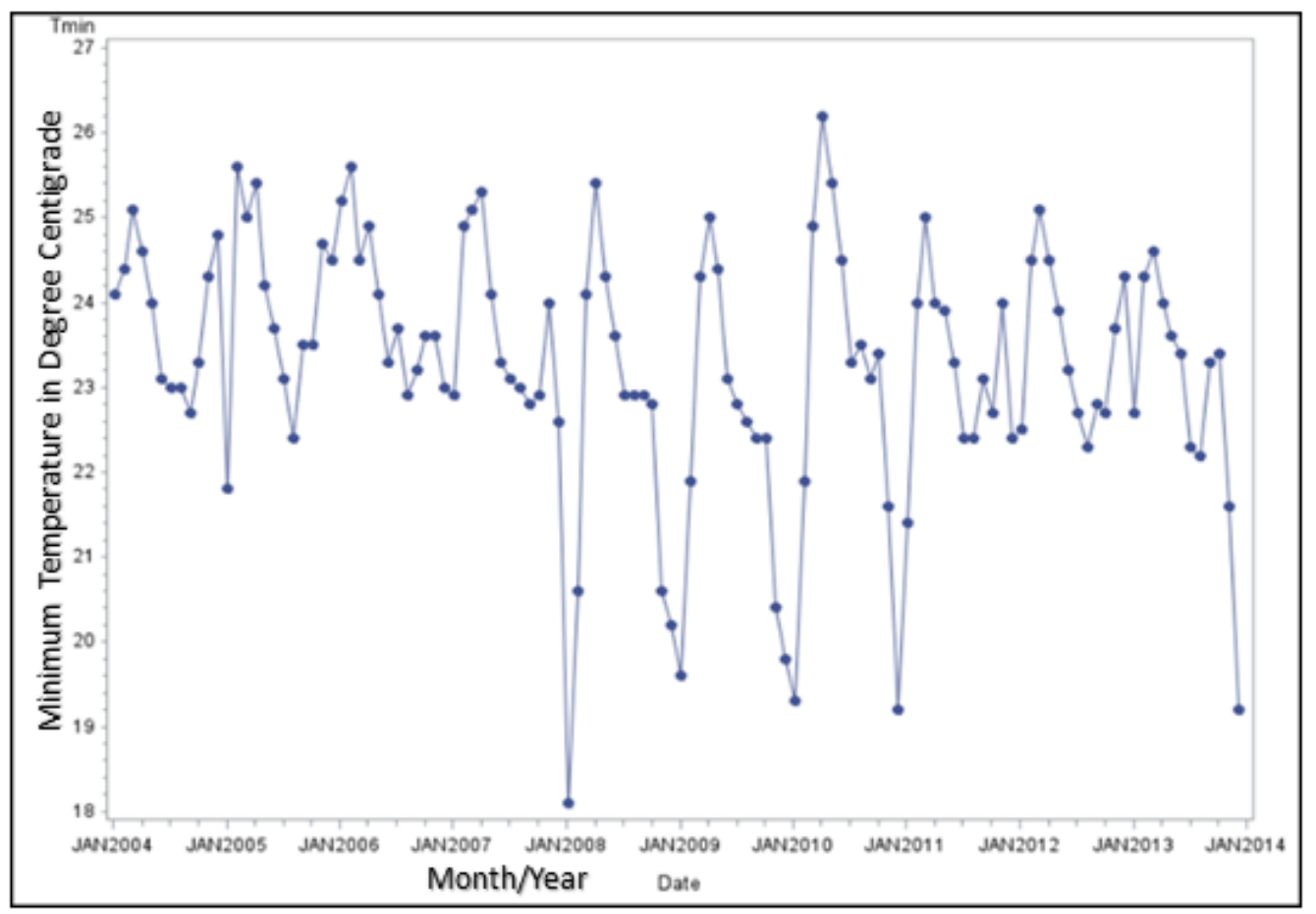

Figure 7. Monthly minimum temperature in degree centigrade between January 2004 and December 2013

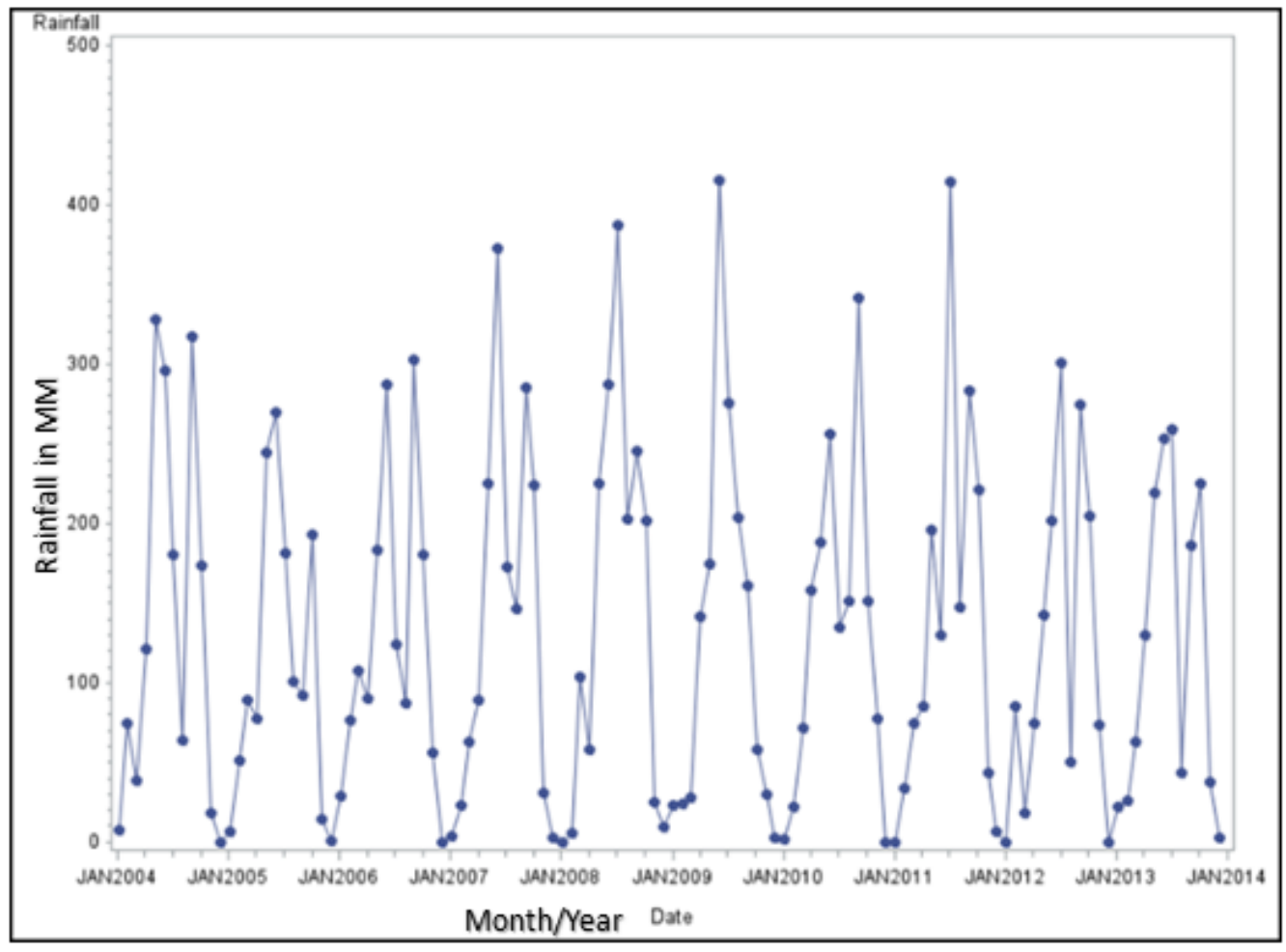

Figure 8. Monthly rainfall in mm between January 2004 and December 2013 


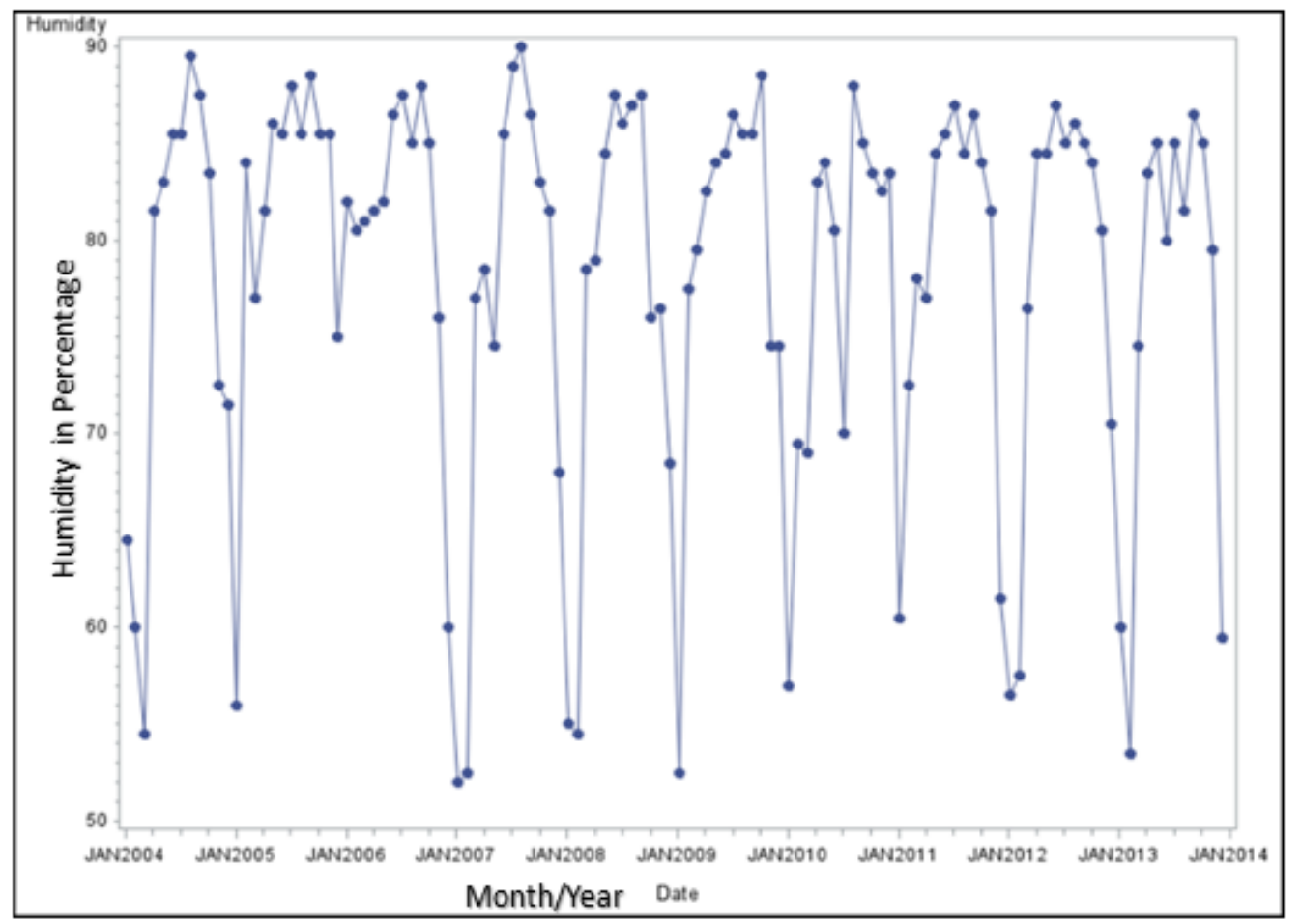

Figure 9. Monthly relative humidity in percentage between January 2004 and December 2013

\subsection{Rainfall}

The average rainfall was $128.9 \mathrm{~mm}$. The rainfall ranges from $0 \mathrm{~mm}$ to $415.6 \mathrm{~mm}$ in June, 2009. Fig. 8 shows the variation of the monthly rainfallin Ogun State from January 2004 to December 2013.

\subsection{Relative Humidity}

The average relative humidity was 78.3 percent. The relative humidity ranges from $52 \%$ in January, 2007 to $90 \%$ in August, 2007. Some of the lowest humidity were recorded in the months and years of January, 2007 (52\%), January, 2009 (52.5\%), February, 2007 (52.5\%), February, $2013(53.5 \%)$ and February, $2008(54.5 \%)$ and the highest relative humidity were recorded in the months and years of September, 2005 (88.5\%), October, 2009 (88.5\%), July, 2007 (89\%), August, 2004 (89.5\%) and August, 2007 $(90 \%)$. Figure 9 shows the variation of the monthly relative humidity in Ogun State from January 2004 to December 2013.

\subsection{Model 1: Malaria Cases $=$ Rainfall}

Table 1 presents the parameter estimates for model 1 which includes the log malaria cases as the dependent variable and rainfall as the independent variable. In this model, the value of the coefficient for rainfall was $($ exponential of 0.000935$)=1.00093)$ suggests that the malaria count for pregnant women in Ogun State increases by $0.1 \%$ for each $\mathrm{mm}$ increase in rainfall. This coefficient is statistically significant. The value of the constant (intercept) was 555.184. This is the count of malaria cases when all of the predictors are equal to zero. The estimate for alpha is 1.178 . For comparison, a model with an alpha of zero is equivalent to a zero-truncated Poisson model. In this model, alpha is statistically different from zero, suggesting that the negative binomial model is a better choice than a Poisson model.

\subsection{Model 2: Malaria Cases $=$ Humidity}

Table 2 presents the parameter estimates for model 2 which included the log malaria cases as the dependent variable and humidity as the independent variable. In this model, the value of the coefficient for humidity is (exponential of 0.007698$)=1.008$ ) suggests that the malaria count for pregnant women in Ogun State increases by $0.8 \%$ for each percent increase in relative humidity. This coefficient is statistically significant. The value of the constant (intercept) is 343.883 . This is the count of malaria cases when all of the predictors are equal to zero. The estimate for alpha is 1.183 . For comparison, a model with an alpha of zero is equivalent to a zero-truncated Poisson model. In this model, alpha is statistically different from zero, suggesting that the negative binomial model is a better choice than a Poisson model. 
Table 1. Parameter Estimates for Log Transformed malaria cases and Rainfall.

\begin{tabular}{|c|c|c|c|c|c|c|c|c|c|c|}
\hline & \multicolumn{10}{|c|}{ Parameter Estimates } \\
\hline Parameter & Estimate & $\begin{array}{c}\text { Standard } \\
\text { Error }\end{array}$ & $\begin{array}{c}\text { EXP } \\
\text { Estimate }\end{array}$ & DF & T Value & Pr $>|t|$ & Alpha & Lower & Upper & Gradient \\
\hline intercept & 6.3193 & 0.05778 & 555.184 & 120 & 109.37 & $<.0001$ & 0.05 & 6.2049 & 6.4337 & 0.000768 \\
\hline Rainfall & 0.000935 & 0.000341 & 1.001 & 120 & 2.75 & 0.0070 & 0.05 & 0.00026 & 0.00161 & -0.02847 \\
\hline alpha & 0.1636 & 0.02081 & 1.178 & 120 & 7.86 & $<.0001$ & 0.05 & 0.1224 & 0.2048 & -0.00087 \\
\hline
\end{tabular}

Table 2. Parameter Estimates for Log Transformed malaria cases and Humidity.

\begin{tabular}{|c|c|c|c|c|c|c|c|c|c|c|c|}
\hline & \multicolumn{10}{|c|}{ Parameter Estimates } \\
\hline Parameter & Estimate & $\begin{array}{c}\text { Standard } \\
\text { Error }\end{array}$ & $\begin{array}{c}\text { EXP } \\
\text { Estimate }\end{array}$ & DF & t Value & $\operatorname{Pr}>|t|$ & Alpha & Lower & Upper & Gradient \\
\hline intercept & 5.8403 & 0.2856 & 343.883 & 120 & 20.45 & $<.0001$ & 0.05 & 5.2748 & 6.4059 & $8.677 \mathrm{E}-7$ \\
\hline Humidity & 0.007698 & 0.003616 & 1.008 & 120 & 2.13 & 0.0353 & 0.05 & 0.00054 & 0.01486 & -0.00027 \\
\hline alpha & 0.1678 & 0.02132 & 1.183 & 120 & 7.87 & $<.0001$ & 0.05 & 0.1256 & 0.2100 & -0.00009 \\
\hline
\end{tabular}

Table 3. Parameter Estimates for Log Transformed malaria cases and Minimum Temperature.

\begin{tabular}{|c|c|c|c|c|c|c|c|c|c|c|}
\hline & \multicolumn{10}{|c|}{ Parameter Estimates } \\
\hline Parameter & Estimate & $\begin{array}{c}\text { Standard } \\
\text { Error }\end{array}$ & $\begin{array}{c}\text { EXP } \\
\text { Estimate }\end{array}$ & DF & $\mathrm{t}$ Value & $\operatorname{Pr}>|\mathrm{t}|$ & Alpha & Lower & Upper & Gradient \\
\hline intercept & 7.2468 & 0.6058 & 1403.61 & 120 & 11.96 & $<.0001$ & 0.05 & 6.0473 & 8.4464 & 0.000035 \\
\hline MinTemp & -0.03444 & 0.02597 & 0.0966 & 120 & -1.33 & 0.1873 & 0.05 & -0.08586 & 0.01698 & 0.000843 \\
\hline alpha & 0.1712 & 0.02174 & 1.187 & 120 & 7.88 & $<.0001$ & 0.05 & 0.1282 & 0.2143 & -0.00009 \\
\hline
\end{tabular}

Table 4. Parameter Estimates for Log Transformed malaria cases and Maximum Temperature.

\begin{tabular}{|c|c|c|c|c|c|c|c|c|c|c|}
\hline & \multicolumn{10}{|c|}{ Parameter Estimates } \\
\hline Parameter & Estimate & $\begin{array}{c}\text { Standard } \\
\text { Error }\end{array}$ & $\begin{array}{c}\text { EXP } \\
\text { Estimate }\end{array}$ & DF & T Value & Pr $>|t|$ & Alpha & Lower & Upper & Gradient \\
\hline intercept & 7.9383 & 0.5582 & 2802.59 & 120 & 14.22 & $<.0001$ & 0.05 & 6.8331 & 9.0435 & -0.00083 \\
\hline MaxTemp & -0.04628 & 0.01722 & 0.849 & 120 & -2.69 & 0.0082 & 0.05 & -0.08037 & -0.01219 & -0.02803 \\
\hline alpha & 0.1642 & 0.02088 & 1.178 & 120 & 7.86 & $<.0001$ & 0.05 & 0.1228 & 0.2055 & -0.00299 \\
\hline
\end{tabular}

\subsection{Model 3: Malaria Cases $=$ Minimum Temperature}

Table 3 presents the parameter estimates for model 3 which included the log malaria cases as the dependent variable and minimum temperature as the independent variable. In this model, the value of the coefficient for minimum temperature is (exponential of -0.03444) = 0.0966) suggests that the malaria count for pregnant women in Ogun State decreases by $9.7 \%$ for each centigrade increase in minimum temperature. This coefficient is not statistically significant. The value of the constant (intercept) is 1403.61. This is the count of malaria cases when all of the predictors are equal to zero. The estimate for alpha is 1.187. For comparison, a model with an alpha of zero is equivalent to a zero-truncated Poisson model. In this model, alpha is statistically different from zero, suggesting that the negative binomial model is a better choice than a Poisson model.

\subsection{Model 4: Malaria Cases $=$ Maximum Temperature}

Table 4 presents the parameter estimates for model 4 which included the log malaria cases as the dependent variable and maximum temperature as the independent variable. In this model, the value of the coefficient for maximum temperature is (exponential of -0.04628$)=0.849$ ) suggests that the malaria count for pregnant women in Ogun State decreases by $0.8 \%$ for each centigrade increase in maximum temperature. This coefficient is statistically significant. The value of the constant (intercept) is 2802.59. This is the count of malaria cases when all of the predictors are equal to zero. The estimate for alpha is 1.178 . For comparison, a model with an alpha of zero is equivalent to a zero-truncated Poisson model. In this model, alpha is statistically different from zero, suggesting that the negative binomial model is a better choice than a Poisson model. 
Table 5. Parameter Estimates for Log Transformed malaria cases and Maximum Temperature, Minimum Temperature, Humidity and Rainfall.

\begin{tabular}{|c|c|c|c|c|c|c|c|c|c|}
\hline \multicolumn{10}{|c|}{ Parameter Estimates } \\
\hline Parameter & Estimate & Standard Error & DF & $\mathrm{t}$ Value & $\operatorname{Pr}>|\mathrm{t}|$ & Alpha & Lower & Upper & Gradient \\
\hline intercept & 7.3275 & 1.0086 & 120 & 7.26 & $<.0001$ & 0.05 & 5.3304 & 9.3245 & 0.000189 \\
\hline MaxTemp & -0.00655 & 0.02630 & 120 & -0.25 & 0.8038 & 0.05 & -0.05862 & 0.04553 & 0.006334 \\
\hline MinTemp & -0.04616 & 0.02729 & 120 & -1.69 & 0.0933 & 0.05 & -0.1002 & 0.007870 & 0.003928 \\
\hline Humidity & 0.003843 & 0.005278 & 120 & 0.73 & 0.4680 & 0.05 & -0.00661 & 0.01429 & 0.00662 \\
\hline Rainfall & 0.000740 & 0.000468 & 120 & 1.58 & 0.1169 & 0.05 & -0.00019 & 0.001667 & -0.05667 \\
\hline alpha & 0.1580 & 0.02013 & 120 & 7.85 & $<.0001$ & 0.05 & 0.1182 & 0.1979 & -0.00111 \\
\hline
\end{tabular}

Table 6. Parameter Estimates for Log Transformed Malaria cases and Rainfall, Humidity and Maximum Temperature

\begin{tabular}{|c|c|c|c|c|c|c|c|c|c|}
\hline \multicolumn{10}{|c|}{ Parameter Estimates } \\
\hline Parameter & Estimate & Standard Error & DF & $\mathrm{t}$ Value & $\operatorname{Pr}>|\mathrm{t}|$ & Alpha & Lower & Upper & Gradient \\
\hline intercept & 7.1605 & 1.0232 & 120 & 7.00 & $<.0001$ & 0.05 & 5.1347 & 9.1863 & -0.00198 \\
\hline MaxTemp & -0.02559 & 0.02433 & 120 & -1.05 & 0.2951 & 0.05 & -0.07377 & 0.02259 & -0.06585 \\
\hline Humidity & 0.000391 & 0.004941 & 120 & 0.08 & 0.9370 & 0.05 & -0.00939 & 0.01017 & -0.14702 \\
\hline Rainfall & 0.000589 & 0.000465 & 120 & 1.27 & 0.2078 & 0.05 & -0.00033 & 0.001510 & -0.13956 \\
\hline alpha & 0.1617 & 0.02058 & 120 & 7.86 & $<.0001$ & 0.05 & 0.1210 & 0.2025 & -0.00144 \\
\hline
\end{tabular}

\subsection{Model 5: Malaria cases $=$ Rainfall + Humidity + Minimum Temperature + Maximum Temperature}

Table 5 presents the parameter estimates for model 5 which included the log malaria cases as the dependent variable and maximum temperature, minimum temperature, rainfall and humidity as the independent variable. In this model, after controlling for all the meteorological variables, none of the variables were statistically significant. The value of the constant (intercept) is 7.3275 the log count of malaria cases when all of the predictors are equal to zero. The estimate for alpha is 0.1580 . For comparison, a model with an alpha of zero is equivalent to a zero-truncated Poisson model. In this model, alpha is statistically different from zero, suggesting that the negative binomial model is a better choice than a Poisson model.

\subsection{Model 6: Malaria cases $=$ Rainfall + Humidity + Maximum Temperature (Significant variables)}

Table 6 presents the parameter estimates for model 6 which included the log malaria cases as the dependent variable and the significant variables which includes maximum temperature, rainfall and humidity as the independent variable. In this model, after controlling for all the significant meteorological variables, none of the variables were statistically significant. The value of the constant (intercept) is 7.1605 the log count of malaria cases when all of the predictors are equal to zero. The estimate for alpha is 0.1617 . For comparison, a model with an alpha of zero is equivalent to a zero-truncated Poisson model. In this model, alpha is statistically different from zero, suggesting that the negative binomial model is a better choice than a Poisson model.

\section{Discussion}

The results of this study indicate that climatic variables as a statistically significant effect on malaria cases among pregnant women in Ogun State, Nigeria. This confirms several studies that have shown a relationship between meteorological variables and malaria incidence in difference population around the world. A study conducted in south-west China in 2008 to determine the effect of weather conditions on the transmission of malaria shows a greater effect of minimum temperature on malaria incidence during cool months than the hot months. Also, there was a positive effect of October fog on the effect of malaria transmission in May of the subsequent year [15]. In a multiplicative seasonal autoregressive integrated moving average (ARIMA) and ARIMAX models to develop best models using data for forecasting and prediction models of malaria incidence in the endemic districts of Bhutan from 1994 to 2006. Different models were developed representing the best fit for both the input and response for each of the endemic areas. These models used both the seasonal and non-seasonal auto regressive and moving average parameters which included $(2,1,1)(0,1,1) 12$, $(2,1,1)(0,1,1) 12$ and $(1,1,1)(0,1,1) 12$. The author concluded that the ARIMAX model of monthly cases and climatic factors showed variations among the different areas and there is positive relationships between the maximum temperatures at lag one and malaria cases in four districts while other two districts showed no relationship [16]. Also, a study to evaluate the association between rainfall, relative humidity, minimum and maximum temperature and malaria in two malaria endemic tropical rain forest areas in south west and north central Nigeria using a monthly malaria data from 2001 to 2007 shows that 
rainfall and humidity had a positive association with malaria incidence at log one month. The others had an inverse association $[17,18]$.

The study has a number of limitations. Since the data used for this study was a secondary data, this study is prone to some of the limitations associated with secondary data usage which includes data quality, data accuracy and less control on the data since the data was collected for different purposes. Some of the cases for some months were missing and in order to minimize the impact of this on the results, a cubic spline interpolation method was used to replace the missing cases. The unavailability of daily or weekly data for malaria cases and meteorological variables rather than a monthly report would have increase the accuracy of the analysis, particularly when climatic conditions were considered over a monthly period. Lastly, socio-economic factors and potential confounding factors such as malaria intervention program instituted by the state which could affect the transmission of malaria were not included in the analysis due to unavailability of data. Zero-truncated negative binomial regression was used, which accurately measures the relationship and addresses the underlying concerns of normal Poisson regression, which ultimately controls for errors and bias in our result.

\section{Conclusions}

This study shows that there is a relationship between climatic conditions and malaria cases among pregnant women in Ogun State, Nigeria. Although little or nothing can be done to stop climatic conditions, more aggressive malaria preventive measures should be instituted to protect pregnant women during adverse weather conditions.

\section{Acknowledgements}

We acknowledge the support of Ms. Adeyinka, the malaria program coordinator for Ogun State, Mr. Adeyemi, the Data Analyst for the malaria program in Ogun State and Mr. Joseph Alozie, General Manager Climate Services, Nigerian Metrological Agency (NiMet) for their tireless effort in making sure that this research is a monumental success. Special thanks to the Florida A\&M University Institute of Public Health for giving us the opportunity to conduct this research.

\section{REFERENCES}

[1] Monif G, Baker DA: Infectious Disease in Obstetrics and Gynaecology. New York Parthenon. 2004, 286 pp

[2] Desai M, TerKuile FO, Nosten F, McGready A et al.: Epidemiology and burden of malaria in pregnancy. Lancet Infect Dis. 2007, 7:93-104.

[3] World Health Organization: The Africa Malaria Report, 2003.

[4] Ofori MF, Ansah E, Agyepong I: Pregnancy-associated malaria in a rural community of Ghana. Ghana Med J. 2009, 43:13-18.

[5] Nigeria Malaria Fact Sheet: United States Embassy in Nigeria, 2011.

[6] Nigeria Malaria Control Program Strategic Plan 2009-2013: A Road Map for Malaria Control in Nigeria, 2008.

[7] United Nations Children's Educational Fund: Malaria is alive, well \& killing more than 3000 children a day in Africa, 2003.

[8] Roll Back Malaria Partnership: Global Malaria Action Plan II (Action and Investment for Malaria), 2015.

[9] United Nations Children's Fund: Malaria and Children, Progress in intervention coverage: Roll Back Malaria Partnership, 2008.

[10] World Health Organization: Updated WHO Fact Sheet on malaria, 2016.

[11] Ogun State Government: Population Figures, 2014.

[12] Nigeria Galleria: All you need to know about Ogun State, 2014.

[13] Statistical Analysis System: Introduction to SAS: Zero-Truncated Negative Binomial/SAS Data Analysis Examples, 2016.

[14] Statistical Analysis System: SAS/ETS 9.2 User's Guide, 2010.

[15] Tian L, Bi Y, Ho S, Liu W et al.: One-year delayed effect of fog on malaria transmission: a time-series analysis in the rain forest area of Mengla County, south-west China. Malaria Journal. 2008, 7:110.

[16] Wangdi K, Singhasivanon P, Silawan T, and Lawpoolsri S. et al:: Development of temporal modelling for forecasting and prediction of malaria infections using time-series and ARIMAX analyses: A case study in endemic districts of Bhutan. Malaria Journal. 2010, 9:251.

[17] Akinbobola A, Omotosho J: Predicting Malaria Occurrence in Southwest and North central Nigeria using Meteorological parameters. Int J Biometeorol. 2013, 57: 721-728.

[18] Akinbobola A, Omotosho J: Predicting Malaria epidemics in Akure, Southwest, Nigeria using Meteorological parameters. KMS 10 $0^{\text {th }}$ Conference Special Issue, 2013. 